\title{
Geschichte in Köln
}

Zeitschrift für Stadt- und Regionalgeschichte

63

2016

Herausgegeben von

Thomas Deres - Christian Hillen - Michael Kaiser - Martin Kröger

Stefan Lewejohann - Georg Mölich - Joachim Oepen - Wolfgang Rosen Lars Wirtler - Stefan Wunsch

in Verbindung mit

Freunde des Kölnischen Stadtmuseums e.V.

Band 632016

herausgegeben von

Christian Hillen, Joachim Oepen und Stefan Wunsch

\section{ELEKTRONISCHER SONDERDRUCK}

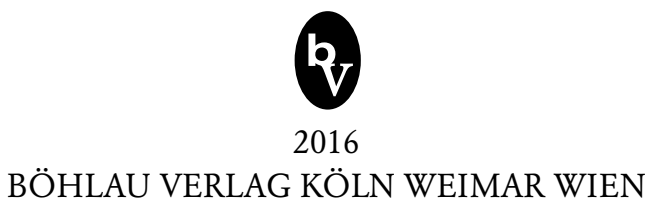




\section{Inhalt}

Britta Hermans "Sanctum eum adprime virum esse«.

Die Vita Brunonis des Ruotger als Bischofsvita

Gerhard-Peter Handschuh Body Snatching, Königsraub oder

Staatsstreich? Die Entführung König Heinrichs IV. in Kaiserswerth:

Der Versuch einer Rekonstruktion

Stefan Pätzold Reinaldus multiplex - oder: Wie viele Hüte passen auf einen Kopf? Zur Persönlichkeit des Kölner Erzbischofs Reinald von Dassel (1159-1167)

Letha Böhringer/Joachim Oepen Wer hat's erfunden?

Ist Rainald von Dassel der "Erfinder« der Dreikönigsreliquien?

Wolfgang Schmid Von den Heiligen Drei Königen zum

Heiligen Rock. Die Formierung der rheinischen Kultlandschaft im 11. und 12. Jahrhundert

Simon Ebert "Das Kölner Polizei-Panama«. Sozialdemokratie, Medien und Skandale im Kaiserreich am Beispiel des Kölner Polizeiprozesses von 1914

Stephanie Kaiser/Jens Lohmeier Aus der Geschichte des Anatomischen Institutes der Universität zu Köln - Die Nutzung Hingerichteter im »Dritten Reich»

Josef van Elten "Fringsen" - Hintergrund und Wirkung einer Predigt des Kölner Erzbischofs Frings

Cedric Bierganns Kultureller Kalter Krieg am Rhein. Das Amerikahaus Köln und der NATO-Doppelbeschluss 


\section{Miszellen}

Thomas Roth Die Gestapo Köln - Ansätze weiterer Forschung. Überlegungen zu einem Projekt des NS-Dokumentationszentrums der Stadt Köln

Christine Gundermann Öffentliche Geschichte -

Public History an der Universität zu Köln

Buchbesprechungen

Carl Dietmar/Werner Jung: Köln. Die große Stadtgeschichte von Clemens von Looz-Corswarem

Udo Mainzer: Kleine illustrierte Kunstgeschichte der Stadt Köln von Anna Pawlik

Mario Kramp: Köln und seine Agrippina. Vom Monster zur Mutter: Zum 2000. Geburtstag der römischen Kaiserin von Lars Wirtler

Raymund Gottschalk: Römer und Franken in Hürth von Lars Wirtler

Norbert Brinkmann: Der Kölner Aufruhr von 1525 im Spiegel der Verhörprotokolle und Dossiers von Clemens von Looz-Corswarem

Uwe Piontek: Kölsch Militär. Legionäre, Spießbürger, Gardisten. 2000 Jahre Soldaten in Köln von Michael Kaiser

Joachim Deeters (Bearb.): Rat und Bürgermeister in Köln 1396-1797.

Ein Verzeichnis von Joachim Oepen

Die evangelischen Kirchenordnungen des XVI. Jahrhunderts. Bd. XXI:

Nordrhein-Westfalen I von Michael Kaiser

Andreas Rutz (Hg.): Krieg und Kriegserfahrung im Westen des Reiches 1568-1714 von Stefan Lewejohann 
Kathrin Kober: Der Kölner Residentenstreit um das exercitium reformatae religionis. Gesandtenrecht versus Staatskirchenrecht zu Anfang des 18. Jahrhunderts von Gerd Schwerhoff

Mario Kramp (Hg.): 125 Jahre Kölnisches Stadtmuseum.

125 mal gekauft - geschenkt - gestiftet von Thomas Deres

Marianne Bechhaus-Gerst/Anne-Kathrin Horstmann (Hg.):

Köln und der Deutsche Kolonialismus. Eine Spurensuche von Markus Kirschbaum

Kathrin Zehender: Christine Teusch. Eine politische Biographie von Winfried Herbers

Renate Goldmann/Erhard Knauer/Eusebius Wirdeier (Hg.):

Moderne. Weltkrieg. Irrenhaus. 1900-1930. Brüche in der Psychiatrie Kunst und Psychiatrie von Thomas Deres

Anette Kunz/Ulrich Mergner: Auf dem Weg zur Disziplin Hundert Jahre getragene Ausbildung für die Soziale Arbeit in Köln 1914-2014 von Martin Oelgeklaus

Winfried Seibert: Die Kölner Kontroverse. Legenden und Fakten um die NS-Verbrechen in Köln-Ehrenfeld Martin Rüther: „Senkrecht stehen bleiben«. Wolfgang Ritzer und die Edelweißpiraten. Unangepasstes Jugendverhalten im Nationalsozialismus und dessen späte Verarbeitung. Mit einem Geleitwort von Werner Jung und Beiträgen von Sonja Schlegel und Irmi von Eckardstein und Opposition im Nationalsozialismus von Kurt Schilde

Mein Leben nach Auschwitz. Erinnerungen von Rachel Grünebaum, aufgeschrieben von Holger Banse und Gabriele Grünebaum von Stefan Wunsch

Abstracts 


\section{Öffentliche Geschichte - Public History an der Universität zu Köln}

\section{von Christine Gundermann}

Im Oktober 2015 begrüßte die Universität zu Köln erstmals zwanzig Studierende am Historischen Institut, die sich im Masterstudiengang Geschichte für die neue Studienrichtung Public History entschieden hatten. Diese Neuerung korrespondierte mit einer etwas weiter zurückliegenden: der Einrichtung einer Juniorprofessur für Public History, die die Autorin dieses Textes seit 2014 vertreten darf. Damit folgt das Historische Institut einem Trend, der sich seit wenigen Jahren in der Bundesrepublik abzeichnet: der Hinwendung zur "öffentlichen" oder »angewandten Geschichte«, so die gängigen Übersetzungen des Terminus "Public History«. Was darunter zu verstehen ist, welche Angebote unseren Studierenden damit zur Verfügung stehen und welche großen Entwicklungslinien dieses junge Forschungs- und Anwendungsfeld aktuell auszeichnen, darüber soll der folgende Text kurz Auskunft geben.

\section{Was ist Public History?}

Diese fundamentale Frage steht seit mehr als vierzig Jahren mit der Entwicklung der ersten "Public History Studies" an der University of California, Santa Barbara und dem ersten Studiengang zur »Applied History and Social Science» an der Carnegie Mellon University, Pittsburgh zur Diskussion in der Geschichtswissenschaft. ${ }^{1}$ Aus historischer Perspektive überrascht es nicht, dass sich die Antwort auf diese Frage je nach Land, Kultur und politisch-gesellschaftlichen Entwicklungen sowie Perspektiven, angestrebten Zielen und Zukunftsvisionen einer nicht ausschließlich universitär betriebenen Geschichtswissenschaft ändert. ${ }^{2}$ In den USA war Public History ursprünglich eine »Bewegung«, unter der jede

1 Simone Rauthe: Public history in den USA und der Bundesrepublik Deutschland, Essen 2001, S. $88 \mathrm{f}$.

2 Vgl.: Irmgard Zündorf: Zeitgeschichte und Public History, Version: 1.0, in: DocupediaZeitgeschichte, 11.02.2010, URL: http://docupedia.de/zg/Zuendorf_public_history_v1_ de_2010 (Zugriff am 15.09.2016) sowie Thomas Cauvin: Public History: A Textbook of practice, New York 2016. 
Form von öffentlicher Geschichtsdarstellung und -vermittlung verstanden wurde, die fern der universitären Wissenschaft und Schulen praktiziert wurde. Robert Kelley, als einer der Begründer der ersten Studienprogramme, hat dies 1978 in seinem wegweisenden und programmatischen Text zusammengefasst: "In its simplest meaning, Public History refers to the employment of historians and the historical method outside of academia: in government, private corporations, the media, historical societies and museums, even in private practice. Public Historians are at work whenever, in their professional capacity, they are part of the public process. An issue needs to be resolved, a policy must be formed, the use of a resource or the direction of an activity must be more effectively planned - and an historian is called upon to bring in the dimension of time: this is Public History. ${ }^{3}$ Die Geschichtsbewegten hatten dabei in den 1970er Jahren kritisiert, dass die universitäre Geschichtswissenschaft ausschließlich forschende Historiker und Historikerinnen hervorbringe, der Kontakt zur Öffentlichkeit jedoch in der modernen, postindustriellen Gesellschaft von großer Wichtigkeit sei. Public History war damit von Anfang an nur ex negativo zur klassischen Geschichtswissenschaft und dem (vermeintlichen) Elfenbeinturm und nicht über spezifische Untersuchungsobjekte oder methodische Zugänge konzeptualisiert worden. Auch die vom 1980 gegründeten "National Council of Public History (NCPH) der Vereinigten Staaten im Jahr 1992 verabschiedete Kurzbeschreibung von Public History als "[...] history for the public, about the public, and by the public « ${ }^{4}$ betont vor allem den emanzipatorischen Ansatz, der die Ergründung der eigenen Geschichte als Akt der zivilen Mündigkeit in der Gesellschaft hervorhebt.

In der Bundesrepublik war Public History - anders als in den USA - kein Ausbildungs- und Forschungsfeld geworden, aber auch hier entstand Ende der 1970er Jahre eine Geschichtsbewegung. Viele in den USA diskutierte Ansätze wurden auch hier wie in anderen westeuropäischen Ländern diskutiert. Die Alltagsgeschichte, Geschichte von unten, Frauengeschichte oder Erinnerungsgeschichte wurden unter dem Slogan "Grabe, wo du stehst! «" ebenso »entdeckt» und erarbeitet - aber nicht unter dem Begriff der Public History, sondern unter

3 Robert Kelley: Public History: Its Origins, Nature, and Prospects, in: The Public Historian 1 (1978) Fall, S. 16-28, hier S. 16.

4 Charles C. Cole Jr.: Public History: What Difference Has it Made?, in: The Public Historian 16 (1994) 4, S. 9-35, hier S. 11.

5 Nach dem gleichnamigen Buch von Sven Lindquist (1978), das aber erst 1985 in deutscher Übersetzung erschien und in Schweden und anderen europäischen Ländern als Grundlagenwerk der Laienarchäologie und anderer Spielarten der Geschichtsbewegungen galt. Vgl. Gerhard Paul/Bernhard Schoßig (Hg.): Die andere Geschichte. Geschichte von unten. Spurensicherung. Ökologische Geschichte. Geschichtswerkstätten, Köln 1986, S. 23. 
dem der Geschichtsbewegung. Neue inhaltliche Fragen wurden hier vor allem aus der sogenannten Werkstattbewegung heraus an die Geschichte gestellt. Die Geschichtswerkstätten hatten es sich vor allem zur Aufgabe gemacht, die bisher von der akademischen Geschichtsforschung vernachlässigten Gruppen in historischer Perspektive sichtbar zu machen; die (oftmals) eigene soziale Vergangenheit geriet in den Fokus. ${ }^{6}$ Die deutschen Geschichtsbewegten nutzten dabei ähnliche Methoden wie Public Historians überall in der westlichen Hemisphäre. ${ }^{7}$ Die Frage, wer für wen warum Geschichte macht, gewinnt durch den anhaltenden Geschichtsboom seit den 1980er Jahren zunehmend an Gewicht.

Gerade weil in der Bundesrepublik diese Welle einer neuen öffentlichen Geschichte nicht aus den Universitäten heraus diskutiert, sondern an sie herangetragen wurde, ${ }^{8}$ entstanden hier keine Neuausrichtungen des Geschichtsstudiums, durch die Studierende auf einen Arbeitsmarkt jenseits von Schule und (universitärer) Forschung vorbereitet werden sollten. Das hat sich nun geändert: Die Public History und ihre Ausprägungen werden in der Fachliteratur präsenter ${ }^{9}$ und in den deutschen Forschungslandschaften sind neue Lehrstühle mit neuen Denominationen entstanden. Geschichtskultur und Erinnerungskultur sind zu Leitbegriffen dieser »öffentlichen Geschichte« in der Bundesrepublik geworden. ${ }^{10}$

6 Etta Grotrian: Geschichtswerkstätten und alternative Geschichtspraxis in den achtziger Jahren, in: Wolfgang Hardtwig/Alexander Schug (Hg.): History Sells! Angewandte Geschichte als Wissenschaft und Markt, Stuttgart 2009, S. 243-253, hier S. 244.

7 Grotian: Geschichtswerkstätten (Anm. 6); aus historischer Perspektive Gerhard Paul/Bernhard Schoßig (Hg.): Die andere Geschichte. Geschichte von unten. Spurensicherung. Ökologische Geschichte. Geschichtswerkstätten, Köln 1986.

8 Grotrian: Geschichtswerkstätten (Anm. 6) beschreibt in ihrem Beitrag, wie gerade das Forschungsfeld der Alltagsgeschichte, welches durch die Geschichtswerkstätten an die universitäre Lehre und Forschung herangetragen wurde, kritisch beim 35. deutschen Historikertag 1984 in Berlin unter dem Titel "Was kommt nach der Alltagsgeschichte" aufgegriffen wurde. Sie zitiert das Bonmot, wonach Hans-Ulrich Wehler die Geschichtsbewegten heftig wegen ihres mehr und weniger reflektierten Forschungszugriffs als "alternativkulturellen Barfußhistoriker" kritisierte.

9 Stellvertretend: Frank Bösch/Constantin Goschler (Hg.): Public History. Öffentliche Darstellungen des Nationalsozialismus jenseits der Geschichtswissenschaft, Frankfurt am Main 2009 und Wolfgang Hardtwig/Alexander Schug: History sells! (Anm. 6).

10 Christoph Cornelißen: Was heißt Erinnerungskultur? Begriff - Methoden - Perspektiven, in: Geschichte in Wissenschaft und Unterricht 54 (2003), S. 548-563; Moritz Czáky/Peter Stachel (Hg.): Mehrdeutigkeit. Die Ambivalenz von Gedächtnis und Erinnerung, Wien 2002; Marko Demantowsky: Geschichtskultur und Erinnerungskultur - zwei Konzeptionen des einen Gegenstandes, in: Geschichte, Politik und ihre Didaktik 33 (2005) 1-2, S. 11-20; Wolfgang Hardtwig: Deutsche Geschichtskultur im 19. und 20. Jahrhundert, München 2013; Vadim Oswalt/Hans-Jürgen Pandel (Hg.): Geschichtskultur. Die Anwesenheit von Vergangenheit in der Gegenwart, Schwalbach/Ts. 2009; Jörn Rüsen: Auf dem Weg zu einer Pragmatik der Geschichtskultur, in: Ulrich Baumgärtner/Waltraud Schreiber (Hg.): Geschichts-Erzählungen und Geschichts-Kultur. Zwei geschichtsdidaktische Leitbegriffe in der Diskussion, München 2001, S. 81-97; Jörn Rüsen: Die fünf Dimensionen der Geschichtskultur, in: Jacqueline Nießer/Juliane Tomann (Hg.): Angewandte Geschichte. Neue Perspektiven auf Geschichte in der Offentlichkeit, Paderborn 2014, S. 46-57; Bernd Schönemann: Erinnerungskultur 
Das erste Masterprogramm in Public History wurde 2008 von der Freien Universität Berlin angeboten, die erste Professur 2012 in Heidelberg eingerichtet.

Public History ist dennoch keine "normale « Subdisziplin der Geschichtswissenschaft geworden; gerade weil sie den zukünftigen Arbeitsmarkt von Historikern und Historikerinnen nicht nur als Forschungsfeld betrachtet, ist sie immer auch ein Anwendungsfeld, in diesem Sinne wird sie auch teilweise synonym als »angewandte Geschichte « bezeichnet. ${ }^{11}$ Dieses Verständnis zeigt sich nicht zuletzt in der Aufstellung der 2012 gegründeten "Arbeitsgruppe Angewandte Geschichte/Public History « unter dem Dach des Verbands der Historiker und Historikerinnen Deutschlands (VHD). Die damit verbundene Unschärfe spiegelt sich auch in der Verortung der bis jetzt eingerichteten Professuren und Studiengänge wider: Ist Public History ein Feld der neueren und neuesten Geschichte (Heidelberg, Berlin), soll es an der Schnittstelle von Geschichtsdidaktik und anderen epochalen Vertiefungen liegen (Köln), ist es ein Teilgebiet der Geschichtsdidaktik (Flensburg, Bochum und München) oder ist dies ein Feld, das eher der Pädagogik zuzurechnen ist (Hamburg)?

Phänomene der Geschichtskultur wurden dabei in den deutschen Forschungslandschaften schon lange und ausgiebig erforscht, wobei die Studien in großem Maße von "turns" wie dem "cultural turn" und dem großen Feld der »memory studies«, dem "visual turn $\aleph^{12}$ und immer häufiger auch dem "performative turn $\aleph^{13}$ angesiedelt sind. ${ }^{14}$ Wenn aber der NCPH im Jahr 2006 in seiner neuen Definition

oder Geschichtskultur?, in: Eugen Kotte (Hg.): Kulturwissenschaften und Geschichtsdidaktik, München 2011, S. 53-72; international jüngst: Mario Carratero/Stefan Berger/Maria Grever (Hg.): Palgrave Handbook of Research in Historical Culture and Education, Basingstoke 2016.

11 Siehe dazu: Jacqueline Nießer und Juliane Tomann (Hg.): Angewandte Geschichte. Neue Perspektiven auf Geschichte in der Offentlichkeit, Paderborn 2014.

12 Z.B. durch Studien zur Geschichte im Fernsehen wie Rainer Wirtz: Alles authentisch: so warss. Geschichte im Fernsehen oder TV-History, in: Thomas Fischer/Rainer Wirtz (Hg.): Alles authentisch? Popularisierung der Geschichte im Fernsehen, Konstanz 2008, S. 9-32; Beate Schlanstein: Geschichtsdokumentationen im Fernsehen - einige Beobachtungen, in: Martina Padberg/Martin Schmidt (Hg.): Magie der Geschichte. Geschichtskultur und Museum, Bielefeld 2010, S. 63-68; Günter Riederer: Film und Geschichtswissenschaft. Zum aktuellen Verhältnis einer schwierigen Beziehung, in: Gerhard Paul (Hg.): Visual History. Ein Studienbuch, Göttingen 2008, S. 96-113.

13 Im Bereich der Living History Sabine Schindler: Authentizität und Inszenierung. Die Vermittlung von Geschichte an amerikanischen historic sites, Heidelberg 2003; Wolfgang Hochbruck: Geschichtstheater. Formen der "Living History«. Eine Typologie, Bielefeld 2013.

14 Wie Frank Bösch: Ereignisse, Performanz und Medien in historischer Perspektive, in: Frank Bösch/Patrick Schmidt (Hg.): Medialisierte Ereignisse. Performanz, Inszenierung und Medien seit dem 18. Jahrhundert, Frankfurt am Main 2010, S. 7-29; Dietlind Hüchtker: Geschichte als Performanz. Politische Bewegungen in Galizien um 1900, Frankfurt am Main 2014 und einführend Jürgen Martschukat: Geschichtswissenschaft und "performative turn«: Eine Einführung in Fragestellungen, Konzepte und Literatur, in: Jürgen Martschukat (Hg.): Geschichtswissenschaft und "performative turn«: Ritual, Inszenierung und Performanz vom Mittelalter bis zur Neuzeit, Köln 2003, S. 1-32. 
Public History als »movement, methodology, and approach« beschreibt, so ist dies für die Vereinigten Staaten und in jedem Fall für die Bundesrepublik nur bedingt zutreffend, wie auch in den USA kritisch diskutiert wurde. ${ }^{15}$ Die Public History weist heute nationale und internationale (wenn auch noch junge) Strukturen auf, die deutlich für eine Institutionalisierung und nicht länger für eine Bewegung sprechen. Ein methodologischer Kanon ist trotz des klaren Einflusses des "cultural turns« auch heute immer noch nicht ersichtlich - gerade im internationalen Feld zeigt sich zudem, wie deutlich der Einfluss von nationalen Wissenschaftskulturen diese Diskurse bestimmt.

Frank Bösch und Constantin Goschler haben daher als Auftakt der deutschen Diskussion vorgeschlagen, Public History als »jede Form von öffentlicher Geschichtsdarstellung [zu begreifen], die außerhalb von wissenschaftlichen Institutionen, Versammlungen oder Publikationen aufgebracht wird.$^{16}$ Auch Irmgard Zündorf übernahm in ihrem Grundlagenartikel "Zeitgeschichte und Public History ${ }^{17}$ auf der Online-Plattform Docupedia im Jahr 2010 diese Definition, ${ }^{18}$ aktualisierte sie jedoch 2016 entscheidend und bezog die Forschung deutlicher mit ein. ${ }^{19}$

Habbo Knoch betont in seinem Vorschlag die Forschungsperspektive stark und versteht Public History "als Teildisziplin der Geschichtswissenschaft, die öffentliche Repräsentation von Vergangenheit außerhalb von Fachwissenschaft, Schule und Familie $[\ldots]$ untersucht $[\ldots] \ll .{ }^{20} \mathrm{Ob}$ die starke Trennung von Öffentlichkeit und Fachwissenschaft, die die Diskurse um Public History seit den 1970er Jahren begleitet, heute noch sinnvoll ist, muss weiter diskutiert werden. Einerseits werden die Grenzen der akademischen und populären Geschichtsproduktion durchlässiger, andererseits stellen sich akademische Historiker und Historikerinnen durch eine solche Formulierung gleich einer Art akzidenteller

15 Kathy Corbett/Dick Miller: What's in a Name? Kritik an der Definition des National Council on Public History, H-Public Discussion Networks, May 2007, URL: http://h-net. msu.edu/cgi-bin/logbrowse.pl?trx $=$ vx\&list $=H$-Public\&month $=0705 \& w e e k=c \& m s g=H A U u$ HywQGvciGXBxeGKPgw\&user=\&pw= (Zugriff am 15. September 2016).

16 Frank Bösch/Constantin Goschler: Der Nationalsozialismus und die deutsche Public History, in: Frank Bösch/Constantin Goschler (Hg.): Public History. Öffentliche Darstellungen des Nationalsozialismus jenseits der Geschichtswissenschaft, Frankfurt am Main 2009, S. 7-23, S. 10.

17 Zündorf: Zeitgeschichte (Anm. 2).

18 Ebd.

19 Irmgard Zündorf: Zeitgeschichte und Public History, Version: 2.0, in: Docupedia-Zeitgeschichte, 06.09.2016, URL: http://docupedia.de/zg/Zuendorf_public_history_v2_de_2016 (Zugriff am 15.09.2016).

20 Habbo Knoch: Wem gehört die Geschichte? Aufgaben der »Public History« als wissenschaftlicher Disziplin, in: Wolfgang Hasberg/Holger Thünemann (Hg.): Geschichtsdidaktik in der Diskussion. Grundlagen und Perspektiven, Frankfurt am Main 2016, S. 303-346, S. 304. 
Verursachung ins (öffentliche) Abseits - mit der Einrichtung der Public History als Ausbildungsfach ist diese Trennung mit all ihren Konsequenzen meines Erachtens daher nur noch schwer aufrecht zu erhalten.

Einigkeit besteht aber darin, dass keine thematischen und epochalen Grenzen die Public History bestimmen - weder ist sie auf den Umgang mit dem Nationalsozialismus festgelegt, noch auf spezifische Medien und damit auch nicht auf Methoden. Die thematische Eingrenzung der Arbeitsfelder von Public History obliegt also der Öffentlichkeit und ist damit sehr wandelbar - nur was auf ein breites öffentliches Interesse stößt, ist auch ein potentielles Arbeitsfeld für Public Historians ${ }^{21}$ - dies betrifft jedoch nicht den Bereich der Forschung. Gerade hier zeigt sich, dass die universitäre Public History an den Schnittstellen von gängigen Spezialisierungen der Fachwissenschaft einerseits und der Geschichtsdidaktik andererseits (an der Universität zu Köln) einen sehr guten Platz gefunden hat. Nur so kann sie auf den in außerschulischen Kontexten gern genutzen Vermittlungsbegriff kritisch reflektieren und Theorien und Modelle des Historischen Lernens in das eigene theoretische Fundament integrieren. Auch bietet die Geschichtsdidaktik wertvolle Erfahrungen im Bereich der Methoden quantitativer und qualitativer Rezeptionsforschung. ${ }^{22}$

Die Erforschung und Produktion von geschichtskulturellen Phänomenen - als hier vorgeschlagene Minimaldefinition von Public History - ist immer auch ein Überschreiten der Grenzen der Geschichtswissenschaft; die Medien des Historischen folgen ihren eigenen Logiken und werden wiederum nicht nur von Spezialisten und Spezialistinnen erforscht, sondern auch von Spezialwissenschaften untersucht. Darüber hinaus können Wirkungen und Funktionsmechanismen von Geschichtskulturen als Teil der Aneignung und Konstruktion von Welt und Gesellschaft nicht ohne Nachbardisziplinen wie der Ethnologie oder Soziologie verstanden werden. Public History als Subdisziplin der Geschichtswissenschaft muss also immer auch bis zu einem gewissen Grad transdiziplinär gedacht und diskutiert werden - sonst kann sie ihrem Forschungsauftrag nicht gerecht werden und kein ausreichend differenziertes methodisches Instrumentarium zur empirischen Forschung entwickeln. ${ }^{23}$

21 Zündorf: Zeitgeschichte, Version 2.0 (Anm. 18).

22 Holger Thünemann: Public History - 9 Thesen, eine Replik auf Marko Demantowsky: »Public History - Sublation of a German Debate? In: Public History Weekly 3 (2015) 2, DOI: dx.doi. org/10.1515/phw-2015-3292 (Zugriff am 15. September 2016).

23 Vgl. Jörg Rogge: Historische Kulturwissenschaften. Eine Zusammenfassung der Beiträge und konzeptionelle Überlegungen, in: Jan Kusber u.a. (Hg.): Historische Kulturwissenschaften. Positionen, Praktiken und Perspektiven, Bielefeld 2010, S. 351-379. 


\section{Die Studienrichtung Public History an der Universität zu Köln}

Studierende an der Universität zu Köln, die sich im Rahmen ihres MasterGeschichtsstudiums für die Studienrichtung Public History entschieden haben, beginnen jeweils im Wintersemester ihr Studium, welches in insgesamt acht Module gegliedert ist und vier Semester umfasst. Im Vordergrund steht die Analyse und Reflexion des öffentlichen Gebrauchs von Geschichte. Die Studienrichtung verbindet dabei drei Schwerpunkte: Erstens bietet sie allen Studierenden eine fundierte Ausbildung als Historiker und Historikerinnen an - die wissenschaftlichen Grundlagen des Faches stehen also auch hier wie im "normalen" Geschichtsstudium an erster Stelle. Das geschieht jedoch mit dem deutlichen Fokus auf Geschichtskulturen. Dieser Zugang erschließt sich durch eine Einführung und intensive Diskussionen aktueller nationaler und internationaler Konzeptionen der Public History, die die Grundlage des Studiums im ersten Semester bilden. Diese werden von zwei klassischen Forschungsmodulen flankiert, wobei eines verpflichtend in der Neuzeit angesiedelt ist und das zweite nach eigenen Interessen mit einem anderen epochalen und regionalen Schwerpunkt gewählt werden kann.

Auf diesem Fundament bauen die zwei weiteren Säulen des Studiums auf, die (erste) eigene praktische Erfahrungen im Bereich der Public History und die Reflexion auf unterschiedliche Formen öffentlicher Geschichtsrepräsentationen umfassen. Die Studierenden werden in einem speziellen Modul mit Themen und Medien öffentlicher Geschichtsrepräsentationen konfrontiert und erwerben so spezifische Analysefertigkeiten im Umgang mit Geschichtskulturen. Dabei werden nicht ausschließlich Phänomene der neueren und Zeitgeschichte untersucht, sondern ebenso solche anderer Epochen, wenn diese in gegenwärtige Geschichtskulturen hineinwirken. Der eigene Weg in die Praxis erfolgt dann nicht nur durch ein dreimonatiges begleitetes Praktikum, sondern wird zudem durch die Einbindung von Praktikerinnen und Praktikern geebnet, die in Form von Lehrveranstaltung und der Umsetzung kleinerer Projekte mit den Studierenden zusammen diese ersten Schritte in eines der möglichen zukünftigen Arbeitsfelder gemeinsam gehen und diese auch kritisch reflektieren. Hierbei profitieren wir in besonderem Maße vom großen Engagement von Mitarbeitern und Mitarbeiterinnen zum Beispiel vom Kölnischen Stadtmuseum, dem Museumsdienst der Stadt Köln, der Friedrich-Ebert-Stiftung in Bonn, dem Historischen Archiv der Stadt Köln, dem NS-Dokumentationszentrum Köln, dem WDR und vielen anderen lokalen und regionalen geschichtskulturellen Institutionen in und um Köln, darunter die NS-Dokumentation Vogelsang, zudem Institutionen, die auch 
im privatwirtschaftlichen Sektor agieren, wie zum Beispiel das Geschichtsbüro Reder, Röseling \& Prüfer. ${ }^{24}$ Die Studienrichtung Public History ist somit im besten Sinne forschungs- und anwendungsorientiert.

Das zweijährige Studium wird auch hier mit einer Masterarbeit abgeschlossen und qualifiziert damit zur Promotion. Uns ist es dabei ein zentrales Anliegen, dass die Studierenden selbständig arbeiten, aber dennoch immer wieder in verschiedenen Lehr- und Arbeitsformen angehalten werden, auch in kleineren Gruppen gemeinsam Projekte zu bearbeiten. Selbständigkeit und Teamwork sind zwei Kompetenzen, die wir stärken wollen. Gleichzeitig bieten wir eine engagierte Beratung und Betreuung während des ganzen Studiums. Das bedeutet nicht nur intensive Kontakte zu den Hochschullehrern und -lehrerinnen und unserem Netzwerk an Public Historians in Köln und dessen Umland; der Erfahrungsraum der Jahrgangsgruppe oder auch "Kohorte« ermöglicht ebenso eine Dynamik, von der die Studierenden profitieren. Das garantieren wir durch einen kleinen aber regelmäßigen Anteil von "geschlossenen« Lehrveranstaltungen, die nur für die jeweilige Jahrgangsgruppe über das viersemestrige Studium hinweg angeboten werden.

Wie bereits angesprochen, weist Public History als Subdisziplin der Geschichtswissenschaft immer auch transdisziplinäre Elemente auf - die Zusammenarbeit mit Mitarbeitenden der gesamten Philosophischen Fakultät, zum Beispiel aus den Theater- und Medienwissenschaften, der Kunstgeschichte, der Kunst- und Kunsttheorie oder der Ethnologie ist uns daher ebenso ein wichtiges Anliegen und wird über einzelne Lectures, aber auch Co-Teachings in spezifischen Lehrveranstaltungen realisiert. Von diesem fachlichen Austausch profitieren nicht nur die Studierenden, sondern alle beteiligten Kollegen und Kolleginnen.

\section{Ein (kleiner) Blick in die Zukunft}

Die Studienrichtung Public History hat damit einen Prozess intensiviert, der im Historischen Institut der Universität zu Köln bereits seit langer Zeit gewünscht und gefördert wurde: die Kooperationen mit Gedenkstätten und Erinnerungsorten, Museen, Archiven, Geschichtsvereinen und Vertretern und Vertreterinnen

24 Bereits jetzt würde es den Umfang des Beitrages sprengen, würden wir allein die überaus kompetenten und freundlichen Führungen durch Gedenkstätten und Museen aufzählen oder die Praktiker und Praktikerinnen nennen, die im Rahmen eines Vortrages an der Universität zu Köln ihren Wirkungsbereich vorgestellt haben, von denen die Studierenden im ersten Studienjahr profitieren durften und ohne deren Mitwirkung ein solch praxisorientiertes Studienprogramm nicht möglich wäre. 
unterschiedlichster Medien der Geschichtskultur. Das bedeutet mittelfristig einerseits eine Intensivierung der Forschungen zur Geschichte in der Öffentlichkeit, andererseits aber auch ein gezieltes Bewegen in die Öffentlichkeit hinein. Die an der Universität zu Köln ausgebildeten Public Historians werden so in die Lage versetzt, besser Geschichte in der Öffentlichkeit zu kommunizieren. Ein Hauptziel der Geschichtsbewegten seit den 1970er Jahren wird damit nun auch aktiv von den deutschen Universitäten verfolgt, wenn auch unter anderen Vorzeichen. ${ }^{25}$ Gleichzeitig professionalisiert die Ausbildung zum und zur Public Historian den Geschichtsmarkt.

Die damit einhergehenden neuen Forschungen zu Geschichtskulturen wiederum werden die Erkenntnisse über die Funktionen und Mechanismen von Geschichte in der Öffentlichkeit weiter vorantreiben. Mindestens drei Felder werden dabei in Zukunft deutlich intensiver erforscht werden:

Die universitäre Institutionalisierung hat der Public History erstmals einen Raum geschaffen, in der theoretisch-methodische Diskurse systematisch und (trans-)national geführt werden können. Damit einher wird eine deutliche Profilbildung der Universitätsstandorte gehen. Hier werden Kernbegriffe wie Geschichtskultur(en), Erinnerungskultur(en) und Authentizität transdisziplinär und unter Vorzeichen verschiedener »turns" diskutiert werden. Eine stärkere Hinwendung zum "material turn« und "performative turn« können hier gerade die geschichtskulturellen Forschungen zur Zeitgeschichte erweitern und damit auch für zukünftige Produktionen von Geschichtskultur neue Sensibilitäten schaffen. Hier profitiert die Public History im Übrigen sehr stark von Kooperationen mit den (universitären) Institutionen der Alten und Mittelalterlichen Geschichte und der Archäologie.

Ein zweiter großer Schwerpunkt bildet sich um die Frage der Wissenspopularisierungen. Public History wird immer öfter mit Popular History gleichgesetzt. Wir wissen jedoch in historischer Perspektive noch sehr wenig über die Strategien und Strukturen von Wissen(schafts)popularisierungen im Bereich der Geschichte. ${ }^{26}$ Forschungen zum großen Bereich der Geschichtsausstellungen und Geschichtsmuseen können hier einen ersten Weg aufzeigen. In diesem Forschungssegment wird es wohl auch in Zukunft um medienspezifische Analysen

25 Ging es früher darum, vor allem für die jenseits der Universitäten arbeitenden Historiker und Historikerinnen ein Netzwerk und eine berufliche Identität zu schaffen, so ist Public History als Studienfach ein Angebot, vor allem die historischen Diskurse in populärkulturellen Kommunikationsräumen mit einem soliden geschichtswissenschaftlichen Fundament zu stützen und zu professionalisieren.

26 Carsten Kretschmann: Wissenschaftspopularisierung - Ansätze und Konzepte, in: Bernd Hüppauf/Peter Weingart (Hg.): Frosch und Frankenstein. Bilder als Medium der Popularisierung von Wissenschaft, Bielefeld 2009, S. 79-89. 
gehen, die aber durch die Rahmung der Public History stärker mit dem Ziel betrieben werden können, medienübergreifend Strukturen und Strategien der Popularisierung aufzuzeigen, zu verstehen und im forschenden wie anwendungsorientierten Sinne fruchtbar für neue Projekte zu machen.

Gleichzeitig berührt dieses Feld einen zentralen und in der Zukunft in jedem Fall weiter zu diskutierende Begriff: das historische Erleben. Die moderne, postmoderne oder auch gegenwärtige Geschichtskultur wird innerhalb und auch außerhalb der geschichtswissenschaftlichen Diskurse häufig und gerne als Erlebniskultur beschrieben oder mit Begriffen wie Eventisierung oder Gamification näher charakterisiert. ${ }^{27}$ Sie beruhen oftmals auf Beobachtungen von aktuellen Phänomenen wie mittlerweile auch in Deutschland populären Reenactments, Ausstellungen mit hohen digitalen und interaktiven Anteilen, den vermehrten Angeboten von Audioguide-Apps und Ähnlichem. Diese Eventkultur wird meist anderen Modi der Aneignung von Geschichtskultur entgegengesetzt, so zum Beispiel einer Phase der Bildung. ${ }^{28}$ In diesen Charakterisierungen von Geschichtskultur werden oftmals Unterhaltung und Erlebnis gleichgesetzt. Diese Ansätze aus der "Erlebnisökonomie« reduzieren jedoch das beschriebene Phänomen zu häufig auf ein simples Reiz-Reaktions-Schema. ${ }^{29}$ Der Erlebnisbegriff selbst muss historisiert werden. Was zu welcher Epoche, Zeit und in welcher Region, Kultur, Klasse oder Schicht unter dem "Erlebnis Geschichte» verstanden wurde und wird, ist starken Veränderungen unterworfen. Diese gilt es weitaus stärker als bisher zu berücksichtigen. Gleichzeitig ist es notwendig, den Erlebnis-Begriff als Element der Aneignung von Geschichtskultur auf seine theoretische und methodische Tragfähigkeit hin zu untersuchen. Wie kann das "geschichtliche Erlebnis" theoretisch jenseits einer Gegenüberstellung von Kognition und Ästhetik (Emotion) gefasst werden, wie kann es konzeptualisiert, wie beschrieben und wie empirisch erforscht werden?

Als junge Subdisziplin der Geschichtswissenschaft muss sich die Public History also deutlich theoretisch und methodisch positionieren - und zwar im Diskurs. Wie die Erfahrungen in den Vereinigten Staaten, Australien oder den Niederlanden gezeigt haben, muss dies nicht zwangsläufig in einen verpflichtenden spezifischen theoretischen und methodischen Kanon münden,

27 Vgl. Wolfgang Hardtwig: Verlust der Geschichte - oder wie unterhaltsam ist die Vergangenheit?, Berlin 2010.

28 Schönemann, Bernd: Geschichtskultur als Forschungskonzept der Geschichtsdidaktik, in: Zeitschrift für Geschichtsdidaktik, 1 (2002), S. 78-86.

29 Kritisch beleuchtet von Monika Popp: Erlebnisforschung neu betrachtet - ein Ansatz zu ihrer räumlichen Kontextualisierung, in: Zeitschrift für Tourismuswissenschaft, 4 (2012) 1, S. $81-100$, S. $81 \mathrm{ff}$. 
oder wie im Fall Deutschlands vielleicht zu erwarten wäre, eine die zentralen Konzepte zur Geschichtskultur und Erinnerungskultur vereinende »große vereinheitlichende Theorie« hervorbringen. Der Terminus »Public History« ist jedoch kein beliebiger; nicht alles wird er als "umbrella term « fassen können. ${ }^{30}$ Dabei zeichnet sich schon jetzt ab, dass die universitäre Public History in der Bundesrepublik weitaus theorielastiger betrieben wird, als dies in anderen Nationen der Fall ist - ein Phänomen, welches sich in erster Linie durch unterschiedliche Wissenschaftskulturen erklären lässt. Dies ist jedoch kein Nachteil, sondern trägt massiv zur Professionalisierung der Public Historians grenzübergreifend bei, zum Beispiel in internationalen Netzwerken wie der "International Federation of Public History«(IFPH), die seit 2014 jährlich eine große Konferenz ausrichtet. ${ }^{31}$

Letztlich verlangt die Ausbildung zum Public Historian auch immer wieder die Reflexionen auf die Frage, welche Rolle Historiker und Historikerinnen in der Gesellschaft übernehmen (wollen)? Diese "Nabelschau" ist in den Vereinigten Staaten ein immanenter Bestandteil der Public History, wie Patricia Mooney-Melvin zusammenfasst: »All historians should remember that they are citizens as well as scholars and they possess some responsibility to the larger civic community. ${ }^{32}$ Patricia Mooney-Melvin verweist auf eine zivilgesellschaftliche Aufgabe der Historiker und Historikerinnen als aufgeklärte Bürger und Bürgerinnen eines Staates. Sie führt uns letztlich wieder zu der Frage nach der Differenzierung von akademischer und öffentlicher Geschichte zurück. Public History als Arbeitsfeld ist damit immer auch ein normatives; geschichtspolitische Debatten im Kleinen wie im Großen zeugen davon. Die verschiedenen Diskurslinien in der Subdisziplin sind also von Anfang an nicht nur interdisziplinär, sondern immer auch transnational angelegt.

30 Vgl. Marko Demantowsky: "Public History« - Sublation of a German Debate?, in: Public History Weekly 3 (2015) 2, DOI: dx.doi.org/10.1515/phw-2015-3292 (Zugriff am 15.09.2016).

31 Siehe Internetpräsenz der International Federation of Public History/ Fédération Internationale pour l'Histoire Publique, URL: http://ifph.hypotheses.org/.

32 Cauvin: Public History (Anm. 2), S. 2. 